\title{
Genzyme: 15 years of cell and gene therapy research
}

\author{
Caroline De Bie \\ Genzyme Corporate Affairs \\ Europe, Ikaroslaan 53 1930, \\ Belgium \\ Tel.: +3227141745 \\ Fax: +3227141747 \\ E-mail: caroline.debie@ \\ genzyme.com
}

\begin{abstract}
Finding solutions for patients with unmet needs is at the heart of Genzyme's innovative medical research. While small molecules and enzyme-replacement therapies have made significant strides in improving patients' quality of life and expectancy, the solutions to many conditions, such as heart disease or CNS diseases, rely on the capacity of the body to regenerate cells. Cell and gene therapy lend themselves well to providing solutions for these illnesses with no current cure, by harnessing the body's natural ability to heal. Promising research in these fields continues to evolve and constitutes a long-term investment in addressing serious unmet medical needs in various therapeutic areas. Over the past 15 years, Genzyme has established itself as a pioneer in shaping the research and commercial application of cell and gene therapy. Genzyme's first commercially available innovative cell therapies - Carticel ${ }^{\circledR}$ and Epicel ${ }^{\circledR}$ - have provided a solid foundation to move into new, cutting-edge areas.
\end{abstract}

One of the world's leading biotechnology companies, Genzyme is dedicated to making a major positive impact on the lives of people with serious diseases. Since 1981, the company has grown from a small start-up to a diversified enterprise with more than 8500 employees in locations spanning the globe with 2005 revenues of US\$2.7 billion.

With many established products and services helping patients in more than 80 countries, Genzyme is a leader in the effort to develop and apply the most advanced technologies in the life sciences. The company's products and services are focused on rare inherited disorders, kidney disease, orthopedics, cancer, transplant and immune diseases and diagnostic testing.

Part of Genzyme's endeavor and commitment to regenerative therapy research has been a longterm investment in technologies, facilities, expertise and partnerships in the field. A total of 40 Genzyme science employees are fully dedicated to gene therapy research and dozens more to improving existing cell therapies. Genzyme's Sidney street facility in Cambridge, MA, USA, which produces cell therapies for thousands of patients each year, is helping increase the company's cell technology manufacturing capacity. The site is also pioneering innovative quality control measures to increase the effectiveness of testing and time to market.

\section{Research \& products Cell therapy}

Cell therapy at Genzyme began in 1994 with the acquisition of Biosurface Technology, a small biotechnology company from Cambridge, MA, USA.
The company had developed an autologous keratinocyte skin graft to treat severe burn victims. In 1989, this product (Epicel ${ }^{\mathrm{TM}}$ ) had become the first cell-based product for tissue repair commercialized in the USA. At the time of the acquisition, a second cell therapy was being developed, an autologous chrondocyte transplantation to treat cartilage injury $\left(\right.$ Carticel $\left.^{\circledR}\right)$. In 1997, Carticel was the first cell-based therapy to secure US FDA approval. Since then, over 10,000 Carticel implants have been completed worldwide. A next-generation product, known as $\mathrm{MACI}^{\circledR}$ in Europe, uses a collagen membrane as a support for chondrocyte implantation. This requires only minimally invasive surgery, therefore decreasing potential vascular damage and recovery time.

Genzyme's other research in cell therapy over the last decade has focussed on next-generation cartilage repair cell therapy, Parkinson's disease and cardiac cell therapy.

\section{Parkinson's disease}

In 2000 Genzyme, in partnership with Diacrin, conducted a Phase II clinical trial of NeuroCell$\mathrm{PD}$, a cell therapy that involved transplanting porcine fetal neural cells in an attempt to replace cells lost to Parkinson's disease. Results of the trial were inconclusive, but provided additional data and expertise to better understand the mechanisms of cell therapy.

\section{Cardiovascular cell therapy}

Millions of people in the world suffer from congestive heart failure and, to date, there are no surgical, medical or mechanical therapies that repair 
Figure 1. Matrix-induced autologous chondrocyte implantation (MACl).

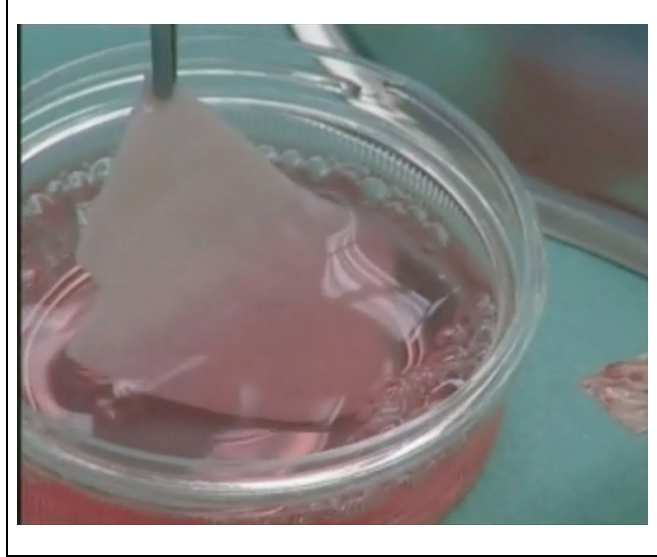

the damaged heart muscle of patients. Interested in addressing this life-threatening condition, Genzyme conducted research investigating whether cultured skeletal muscle cells had the potential to improve cardiac function in infarcted hearts. A Phase II trial initiated in 2002 aimed to demonstrate the efficacy of intramyocardial injection of cultured autologous skeletal myoblasts as a treatment for chronic heart failure. Following the recommendation of the Independent Data Safety Monitoring Board in early 2006, however, enrolment into the trial was ceased, owing to the low likelihood of achieving its primary end point. Patients are currently in follow-up for 1 year.

\section{Gene therapy}

Genzyme initiated research in gene therapy in 1991 with an initial focus on cystic fibrosis (CF). Using its internal expertise in vector technologies, Genzyme launched a clinical trial in which a healthy version of CFRT, the gene that causes CF, was delivered to the lungs of 12 CF patients.

Since then, Genzyme has completed nine clinical trials in $\mathrm{CF}$, testing viral and nonviral vectors, and investing over US $\$ 200$ million on this research. Although no viable treatment has been discovered to date, this experience has allowed Genzyme to investigate other potential applications in a number of fields, such as lysosomal storage disorders, Parkinson's disease, oncology and cardiovascular disease.

\section{Peripheral arterial disease \& cardiac arrhythmia}

The most advanced gene therapy program in Genzyme's pipeline to date is in cardiovascular disease. Genzyme's current research in the field involves introducing a gene that is associated with blood vessel formation, in this case hypoxia inducible factor $(H I F)-1 \alpha$, a 'master gene' discovered at John Hopkins University, Baltimore (MD, USA), with the intention of forming new blood vessels and improving the flow of oxygen to the affected area.

In Genzyme's ongoing Phase II trial, which aims to recruit over 300 patients, a modified version of the $H I F$ gene is injected directly into the lower legs of patients with peripheral arterial disease. The gene is introduced using an adenovirus vector, which escorts the gene into the nucleus of host cells in the leg. Owing to the natural properties of the adenovirus vector, the gene does not integrate into the chromosomes of the cell, and therefore does not alter the genetic makeup of the cell.

HIF-1 $\alpha$ works upstream from vascular endothelial growth factor (VEGF), the protein that promotes blood vessel growth, and other growth factors, and is believed to be the body's own defensive reaction to a low oxygen environment. This solution is also being investigated for patients with coronary artery disease.

\section{Research pipeline}

Considering their potential to offer definite cures for certain illnesses, rather than symptomatic relief or temporary disease treatment, Genzyme is continually investing in and exploring the possibilities of cell and gene therapy. By building partnerships and identifying promising technologies and potential applications, Genzyme is hoping to deliver more solutions to unmet medical needs.

Persevering in its efforts to combat Parkinson's disease, Genzyme is working with investigators at University of California in San Francisco, CA, USA, on the treatment of mid-stage Parkinson's disease. An ongoing 15-patient Phase I/II trial involves introducing an adeno-associated virus $(A A V)$ gene to restore the activity of aromatic L-amino acid decarboxylase (AAAC) in the brain, thereby helping increase the levels of dopamine to reduce the primary symptoms of the disease.

Another area of research is age-related macular degeneration (AMD), in which abnormal blood vessels grow behind the macula, which can lead to damage of the macula and eventually blindness. Preclinical work is currently investigating the potential of introducing a VEGF antagonist molecule using an AAV vector injected in the vitreous portion of the eye. Neutralizing VEGF would slow down or stop the neovascularization occurring in AMD. 


\section{Figure 2. Newly generated blood vessels increase blood flow.}

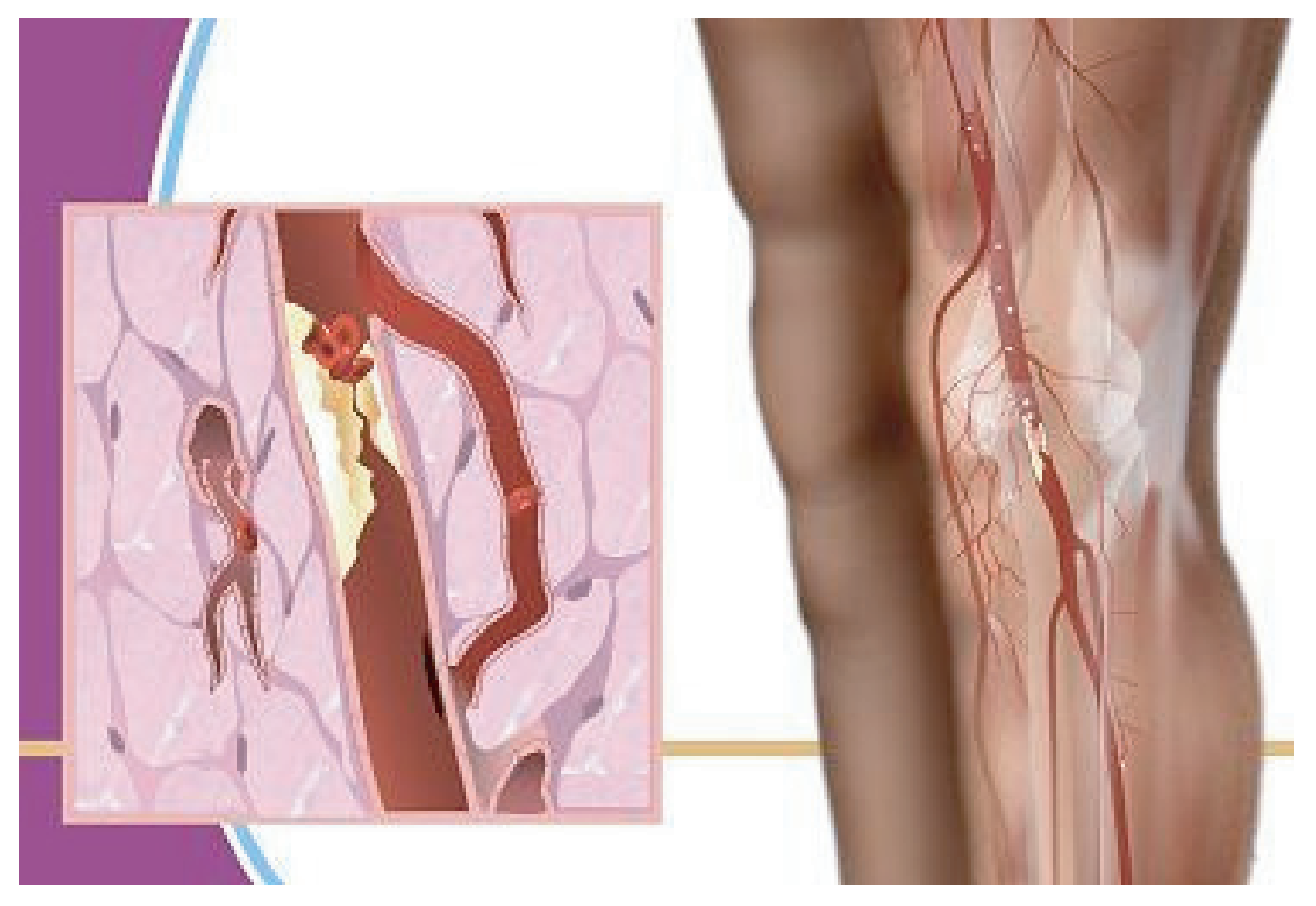

Within the lysosomal storage disorders area, Genzyme is also researching gene therapy solutions for diseases that have a CNS component, such as Niemann Pick A or type 3 Gaucher disease. Several technologies have the potential of delivering genes directly to the brain, getting past the blood-brain barrier.

\section{Outlook}

Genzyme is focused on continuing its innovative cell and gene therapy programs. The future of regenerative medicine is broadly diversified and still largely undetermined, and there are areas uniquely suited to these types of therapies. As with any new medical option, development and adoption of these applications will be guided by a careful weighing of the potential benefits of treatment and the potential risks to patients. Therefore, fully achieving the potential of these new technologies requires a balanced and rigorous regulatory environment that protects the safety of patients while enabling innovation. 